\title{
Hartree-Fock and Random Phase Approximation theories in a many-fermion solvable model
}

\author{
Giampaolo Co:* \\ Dipartimento di Matematica e Fisica "Ennio De Giorgi", \\ Università del Salento, Lecce, \\ and \\ INFN, sez. di Lecce, Italia, \\ Stefano De Leo \\ Departamento de Matemática Aplicada, \\ Universidade Estadual de Campinas, \\ Campinas, Brasil. \\ [Modern Physics Letter A 30, 1550196-15 (2015)]
}

\begin{abstract}
We present an ideal system of interacting fermions where the solutions of the many-body Schrödinger equation can be obtained without making approximations. These exact solutions are used to test the validity of two many-body effective approaches, the Hartree-Fock and the Random Phase Approximation theories. The description of the ground state done by the effective theories improves with increasing number of particles.
\end{abstract}




\section{INTRODUCTION}

The difficulty of solving the Schrödinger equation for quantum many-body systems has induced the development of theories based on approximations which simplify the problem $\underline{\underline{1}} \underline{\underline{\underline{3}}}$. The formalism of these theories is, however, quite involved and its physical content is often overwhelmed by the technical difficulties found in applications to realistic cases.

Simple model systems have been proposed to obtain exact solutions of the Schrödinger equation. These solutions have been used as benchmarks to test the validity of the various approximations in many-body approaches ${ }^{4} \underline{\underline{-8}}$. One of these models has been proposed, in the middle '60s of the last century, by Lipkin, Meshkov, and Glick (LMG) to describe a many-fermion system with two energy levels $\underline{\underline{9}} \underline{\underline{11}}$.

In the spirit of refs. $\stackrel{4}{-\underline{8}}$, after presenting a simple derivation of the exact solutions of the LMG model, we compare these solutions with the results obtained by using two approximation methods. The first one, based on the variational principle, is the Hartree-Fock (HF) theory ${ }^{12}-14$. The second one is the Random Phase Approximation (RPA) which was originally formulated by Bhom and Pines to describe plasma fluctuations $\underline{15} \underline{-18}$.

In section III, we discuss the LMG model and give, when possible, the analytic expressions for the energy levels of the system. In section III, we show how the HF approach can be used to describe the ground state of the system. Then, in section IV, we briefly introduce the RPA theory and calculate the ground-state beyond the HF approximation. Our conclusions are drawn in the final section.

\section{THE LIPKIN MODEL}

In its original version ${ }^{9}$, the LMG model consists of $N$ fermions occupying two energy levels, each of them has an $N$-fold degeneracy. We indicate with $\epsilon$ the energy difference between these two levels. Each level is characterised by a quantum number $\sigma$ which assume the value +1 in the upper level and -1 in the lower one, and by a set $p$ of quantum numbers specifying the particular degenerate states within the same level. Only two-body interactions which scatter pairs of particles between the two levels without changing the value of $p$ are considered. The hamiltonian of this model system is given by

$$
H=\epsilon K_{0}-\frac{V}{2}\left(K_{+}^{2}+K_{-}^{2}\right)-\frac{W}{2}\left(K_{+} K_{-}+K_{-} K_{+}\right),
$$


with

$$
K_{0}=\frac{1}{2} \sum_{p=1}^{N}\left(a_{p,+}^{\dagger} a_{p,+}-a_{p,-}^{\dagger} a_{p,-}\right), \quad K_{+}=\sum_{p=1}^{N} a_{p,+}^{\dagger} a_{p,-} \quad \text { and } \quad K_{-}=K_{+}^{\dagger} .
$$

where $a_{p, \pm}^{\dagger}$ and $a_{p, \pm}$ are the usual fermion creation and annihilation operators satisfying the anti-commutation relations

$$
\left\{a_{p, \alpha}, a_{r, \beta}^{\dagger}\right\}=\delta_{p r} \delta_{\alpha \beta}, \quad\left\{a_{p, \alpha}, a_{r, \beta}\right\}=\left\{a_{p, \alpha}^{\dagger}, a_{r, \beta}^{\dagger}\right\}=0 .
$$

The term proportional to $V$ scatters pair of particles from one level to the other one. The $K_{+}^{2}$ operator removes two particles from the lower level and put them on the upper level. The $K_{-}^{2}$ operator acts in the opposite manner. The term proportional to $W$ promotes a particle in the upper level, creating a hole in the lower level, and, at the same time, removes a particle from the upper level and put it in the lower one.

In the unperturbed situation, i.e. when the interactions are switched off $(V=W=0)$ the energies of the various states are given by the number of particles lying in the upper and lower states, each of them with different values of the quantum number $p$. The lowest energy, that of the ground state, is obtained when all the particles lie on the lower level. Each particle-hole excitation produces a new state whose energy is larger than that of the previous excited state by the quantity $\epsilon$.

We use the properties of the $K_{0, \pm}$ operators to solve the Schrödinger equation for this many-fermion system. By using the anticommutation relations (3), we obtain

$$
\begin{aligned}
{\left[K_{+}, K_{-}\right] } & =\sum_{p, r=1}^{N}\left(a_{p,+}^{\dagger} a_{p,-} a_{r,-}^{\dagger} a_{r,+}-a_{r,-}^{\dagger} a_{r,+} a_{p,+}^{\dagger} a_{p,-}\right) \\
& =\sum_{p, r=1}^{N}\left(a_{p,+}^{\dagger} a_{r,+} \delta_{p r}-a_{r,-}^{\dagger} a_{p,-} \delta_{r p}\right)=2 K_{0}
\end{aligned}
$$

and

$$
\begin{aligned}
{\left[K_{0}, K_{+}\right] } & =\frac{1}{2} \sum_{p, r=1}^{N}\left[\left(a_{p,+}^{\dagger} a_{p,+}-a_{p,-}^{\dagger} a_{p,-}\right) a_{r,+}^{\dagger} a_{r,-}-a_{r,+}^{\dagger} a_{r,-}\left(a_{p,+}^{\dagger} a_{p,+}-a_{p,-}^{\dagger} a_{p,-}\right)\right] \\
& =\frac{1}{2} \sum_{p, r=1}^{N}\left(a_{p,+}^{\dagger} a_{p,+} a_{r,+}^{\dagger} a_{r,-}-a_{r,+}^{\dagger} a_{r,-} a_{p,+}^{\dagger} a_{p,+}+a_{r,+}^{\dagger} a_{r,-} a_{p,-}^{\dagger} a_{p,-}-a_{p,-}^{\dagger} a_{p,-} a_{r,+}^{\dagger} a_{r,-}\right) \\
& =\frac{1}{2} \sum_{p, r=1}^{N}\left(a_{p,+}^{\dagger} a_{r,-} \delta_{p r}+a_{r,+}^{\dagger} a_{p,-} \delta_{r p}\right)=K_{+} .
\end{aligned}
$$


From the last commutation relation, we obtain

$$
\left[K_{0}, K_{-}\right]=-\left[K_{0}, K_{+}\right]^{\dagger}=-K_{-}
$$

The commutation relations (44), (5), and (6) are those of the components of an angular momentum operator in quantum mechanics, therefore all the features related to the angular momentum algebra can be applied to the operators $K_{0, \pm}$. In particular, we observe that the operators $K_{0}$ and

$$
K^{2}=\frac{1}{2}\left\{K_{+}, K_{-}\right\}+K_{0}^{2}
$$

satisfy $\left[K_{0}, K^{2}\right]=0$ and commute with the hamiltonian (11). For these reasons, it is convenient to diagonalise the hamiltonian (1) in the basis of the eigenstates $|k, m\rangle$ of $K^{2}$ and $K_{0}$, whose properties are

$$
\begin{aligned}
K^{2}|k, m\rangle & =k(k+1)|k, m\rangle \\
K_{0}|k, m\rangle & =m|k, m\rangle \\
K_{ \pm}|k, m\rangle & =\sqrt{k(k+1)-m(m \pm 1)}|k, m \pm 1\rangle .
\end{aligned}
$$

We observe that the creation and annihilation structure of the $K_{0}$ operator is such that the $K_{0}$ eigenvalues correspond to count the difference between the particles lying on the upper level and those lying on the lower level and then multiply this difference by a factor $1 / 2$. For example, when all the $N$ particles are in the upper level the eigenvalue of $K_{0}$ is $N / 2$, while when they are all in the lower level, the eigenvalue is $-N / 2$. These are the two extreme values that the eigenvalues of $K_{0}$ can assume. Between these two eigenvalues there is a discrete sequence of eigenvalues each of them differing by a unit, and this produce a total number of $N+1$ states. This is the number of states allowed by the symmetry of the problem, and therefore the dimension of the matrix to be diagonalized to solve the eigenvalue problem.

For a given number $N$ of particles, we solve the Schrödinger equation

$$
H|\Psi, k\rangle=E|\Psi, k\rangle
$$

by expressing the eigenstates $|\Psi, k\rangle$ as linear combination of the $|k m\rangle$ states $^{1}$,

$$
|\Psi, k\rangle=\sum_{m=-\frac{N}{2}}^{\frac{N}{2}} C_{m}|k, m\rangle
$$


The problem of finding the $C_{m}$ coefficients can be expressed in matrix form by bracketing Eq. (9) with $\left\langle k, m^{\prime}\right|$

$$
\sum_{m=-\frac{N}{2}}^{\frac{N}{2}} C_{m}\left\langle k, m^{\prime}|H| k, m\right\rangle=\sum_{m=-\frac{N}{2}}^{\frac{N}{2}} C_{m} E_{k, m}\left\langle k, m^{\prime} \mid k, m\right\rangle=E_{k, m^{\prime}} C_{m^{\prime}}
$$

where in the last step we used the orthonormality of the $|k, m\rangle$ states. In terms of the quantum numbers $m$ and $k$, the matrix elements different from zero are

$$
\begin{aligned}
\langle k, m|H| k, m\rangle & =m \epsilon-W\left[k(k+1)-m^{2}\right], \\
\langle k, m|H| k, m+2\rangle & =-\frac{V}{2} \sqrt{[k(k+1)-m(m-1)][k(k+1)-(m-1)(m-2)]}, \\
\langle k, m+2|H| k, m\rangle & =\langle k, m|H| k, m+2\rangle .
\end{aligned}
$$

For example, the explicit expressions of the hamiltonian matrix and its eigenvalues for the systems composed by two and three particles are

$$
H_{2}=\left(\begin{array}{ccc}
\epsilon-W & 0 & -V \\
0 & -2 W & 0 \\
-V & 0 & -(\epsilon+W)
\end{array}\right), \quad E_{2}=\left\{\begin{array}{l}
+\epsilon \sqrt{1+(V / \epsilon)^{2}}-W \\
-2 W \\
-\epsilon \sqrt{1+(V / \epsilon)^{2}}-W
\end{array},\right.
$$

and

$$
\begin{gathered}
H_{3}=\left(\begin{array}{cccc}
\frac{3}{2}(\epsilon-W) & 0 & -\sqrt{3} V & 0 \\
0 & \frac{1}{2}(\epsilon-7 W) & 0 & -\sqrt{3} V \\
-\sqrt{3} V & 0 & -\frac{1}{2}(\epsilon+7 W) & 0 \\
0 & -\sqrt{3} V & 0 & -\frac{3}{2}(\epsilon+W)
\end{array}\right), \\
2 E_{3}=\left\{\begin{array}{l}
+\epsilon+2 \epsilon \sqrt{1+3(V / \epsilon)^{2}+2(W / \epsilon)+(W / \epsilon)^{2}}-5 W \\
-\epsilon+2 \epsilon \sqrt{1+3(V / \epsilon)^{2}-2(W / \epsilon)+(W / \epsilon)^{2}}-5 W \\
+\epsilon-2 \epsilon \sqrt{1+3(V / \epsilon)^{2}+2(W / \epsilon)+(W / \epsilon)^{2}}-5 W \\
-\epsilon-2 \epsilon \sqrt{1+3(V / \epsilon)^{2}-2(W / \epsilon)+(W / \epsilon)^{2}}-5 W
\end{array}\right.
\end{gathered}
$$

For $W=0$ and $N=4,6,8$ the secular equation is at most quadratic, and exact solutions for the energy eigenvalues can be obtained analytically ${ }^{\underline{9}}$,

$$
\begin{array}{ll}
E_{4} / \epsilon=0, & \pm \sqrt{1+9(V / \epsilon)^{2}}, \quad \pm 2 \sqrt{1+3(V / \epsilon)^{2}}, \\
E_{6} / \epsilon=0, & \pm 2 \sqrt{1+15(V / \epsilon)^{2}}, \quad \pm \sqrt{5+33(V / \epsilon)^{2} \pm 4 \sqrt{1+6(V / \epsilon)^{2}+54(V / \epsilon)^{4}}}, \\
E_{8} / \epsilon=0, & \pm \sqrt{5+113(V / \epsilon)^{2} \pm 4 \sqrt{1+38(V / \epsilon)^{2}+550(V / \epsilon)^{4}}} \\
& \pm \sqrt{10+118(V / \epsilon)^{2} \pm 6 \sqrt{1-2(V / \epsilon)^{2}+225(V / \epsilon)^{4}}} .
\end{array}
$$


The above expressions correct those given in the original paper of Lipkin et al. $\stackrel{9}{ }$ where a factor 4 in $E_{6}$ and 6 in $E_{8}$ are missing.

The green full lines of Fig.1 and Fig. 2 show ground state energy values, $E_{\mathrm{GS}}$, as function of the interaction $V$ for $N=2,3,4$ (Fig.11) and $N=6,8,20$ (Fig.2). In the left panels we show the solutions obtained when $W=0$, and in the right panels those for $W=V$. The results up to $N=8$ have been obtained by using the analytical expressions shown above, while those for $N=20$ by performing a numerical diagonalization of the hamiltonian matrix with standard techniques $\underline{19}$.

The solutions we have presented are exact, meaning that they have been obtained without making approximations of the problem to be solved. These results are our benchmarks to test the validity of the simplified solutions of the many-fermion problems presented in the next sections.

\section{HARTREE-FOCK}

The HF method ${ }^{12-14}$ is one of the most commonly used approaches to describe the ground state of many-fermions systems. The basic HF equations can be obtained in various manners, from the application of the Rietz variational principle ${ }^{\frac{1}{}}$ to the use of the the first order solution of the Dyson equation in the Green's function expansion method ${ }^{2}$. In the present paper we consider the HF approach in its variational formulation.

We search for a ground state solution of the LMG fermion system where all the particles occupy the lower energy level only. This solution is obtained by selecting the low energy level in such a way that the energy of the system is minimal.

We use the basis of states obtained without interaction $(V=W=0)$, then we switch on the interaction and we search for the solution which minimises the total energy of the system. This is equivalent to search for the unitary transformation of the basis of the $|k m\rangle$ states which generates the minimal energy solution.

We obtain a unitary transformation of the $|k m\rangle$ basis by making a unitary trasformation of the creation and annihilation operators

$$
\left[\begin{array}{l}
\tilde{a}_{p,+} \\
\tilde{a}_{p,-}
\end{array}\right]=\left(\begin{array}{rr}
\cos \frac{\alpha}{2} & -\sin \frac{\alpha}{2} \\
\sin \frac{\alpha}{2} & \cos \frac{\alpha}{2}
\end{array}\right)\left[\begin{array}{l}
a_{p,+} \\
a_{p,-}
\end{array}\right],
$$


where the $a$ operators, and they hermitian conjugate, act on the old basis, while the $\tilde{a}$ operators on the new basis. The above transformation defines the new basis in terms of the states of the old basis. In the new basis, we can define the operators

$$
\widetilde{K}_{0}=\frac{1}{2} \sum_{p=1}^{N}\left(\tilde{a}_{p,+}^{\dagger} \tilde{a}_{p,+}-\tilde{a}_{p,-}^{\dagger} \tilde{a}_{p,-}\right), \quad \widetilde{K}_{+}=\sum_{p=1}^{N} \tilde{a}_{p,+}^{\dagger} \tilde{a}_{p,-}, \quad \text { and } \quad \widetilde{K}_{-}=\widetilde{K}_{+}^{\dagger},
$$

which are related to the $K_{0, \pm}$ operators of the old basis by the relation

$$
\left[\begin{array}{c}
\widetilde{K}_{+} \\
\widetilde{K}_{0} \\
\widetilde{K}_{-}
\end{array}\right]=\frac{1}{2}\left(\begin{array}{ccc}
\cos \alpha+1 & 2 \sin \alpha & \cos \alpha-1 \\
-\sin \alpha & 2 \cos \alpha & -\sin \alpha \\
\cos \alpha-1 & 2 \sin \alpha & \cos \alpha+1
\end{array}\right)\left[\begin{array}{c}
K_{+} \\
K_{0} \\
K_{-}
\end{array}\right] .
$$

In analogy with the $K_{0, \pm}$ operators, the new $\widetilde{K}_{0, \pm}$ satisfy the following commutation relations

$$
\left[\widetilde{K}_{+}, \widetilde{K}_{-}\right]=2 \widetilde{K}_{0} \quad \text { and } \quad\left[\widetilde{K}_{0}, \widetilde{K}_{ \pm}\right]= \pm \widetilde{K}_{+} .
$$

We can obtain the expression of the hamiltonian (1) in the new basis by inverting the matrix Eq. (16)

$$
\left[\begin{array}{c}
K_{+} \\
K_{0} \\
K_{-}
\end{array}\right]=\frac{1}{2}\left(\begin{array}{ccc}
\cos \alpha+1 & -2 \sin \alpha & \cos \alpha-1 \\
\sin \alpha & 2 \cos \alpha & \sin \alpha \\
\cos \alpha-1 & -2 \sin \alpha & \cos \alpha+1
\end{array}\right)\left[\begin{array}{c}
\widetilde{K}_{+} \\
\widetilde{K}_{0} \\
\widetilde{K}_{-}
\end{array}\right],
$$

and by inserting the above relations in Eq. (1)

$$
\begin{gathered}
\widetilde{H}=\frac{\epsilon}{2}\left[2 \cos \alpha \widetilde{K}_{0}+\sin \alpha\left(\widetilde{K}_{+}+\widetilde{K}_{-}\right)\right]+\frac{W}{2}\left[\widetilde{K}_{+}^{2}+\widetilde{K}_{-}^{2}-\left\{\widetilde{K}_{+}, \widetilde{K}_{-}\right\}\right] \\
-\frac{V+W}{4}\left[\sin ^{2} \alpha\left(4 \widetilde{K}_{0}^{2}-\left\{\widetilde{K}_{+}, \widetilde{K}_{-}\right\}\right)-\sin 2 \alpha\left(\left\{\widetilde{K}_{0}, \widetilde{K}_{+}\right\}+\left\{\widetilde{K}_{0}, \widetilde{K}_{-}\right\}\right)\right. \\
\left.+\left(1+\cos ^{2} \alpha\right)\left(\widetilde{K}_{+}^{2}+\widetilde{K}_{-}^{2}\right)\right] .
\end{gathered}
$$

To evaluate the expectation value of the hamiltonian Eq. (1) with respect to the new state we make use of the following relations

$$
\begin{aligned}
\widetilde{K}_{0}|\tilde{k}, \widetilde{m}\rangle & =\widetilde{m}|\tilde{k}, \widetilde{m}\rangle, \\
\widetilde{K}_{ \pm}|\tilde{k}, \widetilde{m}\rangle & =\sqrt{\widetilde{k}(\tilde{k}+1)-\widetilde{m}(\widetilde{m} \pm 1)}|\tilde{k}, \widetilde{m} \pm 1\rangle .
\end{aligned}
$$

Therefore, we obtain

$$
\left\langle\tilde{k}, \widetilde{m}\left|\left\{\widetilde{K}_{+}, \widetilde{K}_{-}\right\}\right| \tilde{k}, \widetilde{m}\right\rangle=2\left[\tilde{k}(\tilde{k}+1)-\widetilde{m}^{2}\right]
$$


In the ground state, we have $\tilde{k}=N / 2$ and $\tilde{m}=-N / 2$, consequently

$$
\left\langle\mathrm{HF}\left|\left\{\widetilde{K}+, \widetilde{K}_{-}\right\}\right| \mathrm{HF}\right\rangle=N
$$

The expectation value of the energy in the new basis can be expressed as,

$$
E_{\alpha}=\langle\mathrm{HF}|\widetilde{H}| \mathrm{HF}\rangle=-N \frac{\epsilon}{2}\left[\cos \alpha+\frac{W}{\epsilon}+\frac{(N-1)(V+W)}{2 \epsilon} \sin ^{2} \alpha\right] .
$$

The HF solution is obtained for the values of $\alpha$ which minimize the energy (24), i.e.

$$
\cos \alpha_{\mathrm{HF}}=\left\{\begin{array}{lll}
1 & \text { for } \quad(N-1)(V+W)<\epsilon & \text { (region I) } \\
\frac{\epsilon}{(N-1)(V+W)} & \text { for } \quad(N-1)(V+W)>\epsilon & \text { (region II) }
\end{array}\right.
$$

By using these values, we obtain for the HF energy the expressions

$$
E_{\mathrm{HF}}=-\frac{N}{2}\left\{\begin{array}{lr}
\epsilon+W & \text { (region I) } \\
\frac{\epsilon^{2}+(N-1)^{2}(V+W)^{2}}{2(N-1)(V+W)}+W & \text { (region II). }
\end{array}\right.
$$

The values of the ground state HF energies as a function of the interaction $V$ are shown by the dash-dotted lines of Fig.1 and Fig.2 by dotted lines. For $W=0$ we observe a remarkable difference with the exact solutions, especially in the region I, where the HF energies are constant. In the transition point between the two regions, at $\epsilon=(N-1) V$, the value of the energy is

$$
-\frac{N}{2} \epsilon
$$

For $W=V$ case, we observe a reasonable agreement of the HF solutions with the exact ones, even in the region I. In this case, at the transition point between the two regions, which is located at $\epsilon=2(N-1) V$, the value of the HF energy is

$$
-\frac{N(2 N-1)}{4(N-1)} \epsilon
$$

In the figures, the thick red points indicates these values.

\section{RANDOM PHASE APPROXIMATION}

The second effective theory we consider is the RPA, which was originally formulated to describe the excitations of an electron gas induced by plasma fluctuations 15 , and in the following has been widely applied to describe harmonic vibrations of many-fermion systems 
from atoms to nuclei ${ }^{16} \underline{-18}$. The main goal of the RPA theory is the description of the excited states of the system, but the theory is based on an ansatz about the ground state which is more elaborated than that used in $\mathrm{HF}$.

We present the basic steps required by the RPA theory to obtain an expression of the ground state energy. The starting point is the definition of the operator

$$
Q^{\dagger}=\frac{X \widetilde{K}_{+}-Y \widetilde{K}_{-}}{\sqrt{N}}
$$

which applied to the RPA ground state $|0\rangle$ describe the excited state $|1\rangle$

$$
Q^{\dagger}|0\rangle=|1\rangle
$$

The $Q^{\dagger}$ operator generates a linear combination of one-particle one-hole, and one-hole oneparticle, excitations. In the LMG model this ansatz produces only one excited state which we have identified with $|1\rangle$. The RPA ground state is defined by the equation

$$
Q|0\rangle=0
$$

evidently $|0\rangle$ cannot be $|\mathrm{HF}\rangle$ as the expression (23) indicates. The above definitions and the Schrödinger equation imply

$$
\begin{aligned}
& \widetilde{H} Q^{\dagger}|0\rangle=E_{1}^{\mathrm{RPA}}|1\rangle, \\
& Q^{\dagger} \widetilde{H}|0\rangle=E_{0}^{\mathrm{RPA}} Q^{\dagger}|0\rangle,
\end{aligned}
$$

therefore

$$
\left[\widetilde{H}, Q^{\dagger}\right]|0\rangle=\left(E_{1}^{\mathrm{RPA}}-E_{0}^{\mathrm{RPA}}\right) Q^{\dagger}|0\rangle=\omega Q^{\dagger}|0\rangle
$$

which defines the excitation energy $\omega$. By using the previous equation we write

$$
\begin{aligned}
& \left\langle 0\left|\left[\widetilde{K}_{-},\left[\widetilde{H}, Q^{\dagger}\right]\right]\right| 0\right\rangle=\omega\left\langle 0\left|\left[\widetilde{K}_{-}, Q^{\dagger}\right]\right| 0\right\rangle, \\
& \left\langle 0\left|\left[\widetilde{K}_{+},\left[\widetilde{H}, Q^{\dagger}\right]\right]\right| 0\right\rangle=\omega\left\langle 0\left|\left[\widetilde{K}_{+}, Q^{\dagger}\right]\right| 0\right\rangle .
\end{aligned}
$$

The evaluation of the matrix elements of the above equations is rather difficult since the RPA ground-state defined by Eq. (29) is not defined in terms of particle-hole excitations in our basis. For this reason, the so-called Quasi Boson Approximation (QBA) is used ${ }^{20}$. This approximation consits in substituting all the RPA matrix elements containing commutators 
of particle - hole pair operators with the values obtained by calculating them between HF ground state, i.e.

$$
\left\langle 0\left|\left[a_{h}^{+} a_{p}, a_{p^{\prime}}^{+} a_{h^{\prime}}\right]\right| 0\right\rangle \simeq\left\langle\mathrm{HF}\left|\left[a_{h}^{+} a_{p}, a_{p^{\prime}}^{+} a_{h^{\prime}}\right]\right| \mathrm{HF}\right\rangle=\delta_{p p^{\prime}} \delta_{h h^{\prime}}
$$

The name Quasi Boson Approximation is given since the operator pair $a_{p^{\prime}}^{+} a_{h^{\prime}}$ behaves as a single boson operator. In the specific case under investigation, we have that

$$
\left\langle 0\left|\left[\widetilde{K}_{-}, \widetilde{K}_{+}\right]\right| 0\right\rangle \simeq\left\langle\mathrm{HF}\left|\left[\widetilde{K}_{-}, \widetilde{K}_{+}\right]\right| \mathrm{HF}\right\rangle=-2\left\langle\mathrm{HF}\left|\widetilde{K}_{0}\right| \mathrm{HF}\right\rangle=N,
$$

where we used the commutation relations (17) the relation (20) and the fact that in the HF ground state $|\mathrm{HF}\rangle$ we have $\tilde{m}=-N / 2$. Evidently, the result of Eq. (35) indicates that the $\widetilde{K}_{ \pm}$operators between HF states commute as boson operators.

The RPA master equations Eq. (33) are commonly written by introducing the quantities

$$
\begin{gathered}
A=\frac{\left\langle 0\left|\left[\widetilde{K}_{-},\left[\widetilde{H}, \widetilde{K}_{+}\right]\right]\right| 0\right\rangle}{N} \simeq \frac{\left\langle\mathrm{HF}\left|\left[\widetilde{K}_{-},\left[\widetilde{H}, \widetilde{K}_{+}\right]\right]\right| \mathrm{HF}\right\rangle}{N}, \\
B=-\frac{\left\langle 0\left|\left[\widetilde{K}_{-},\left[\widetilde{H}, \widetilde{K}_{-}\right]\right]\right| 0\right\rangle}{N} \simeq-\frac{\left\langle\mathrm{HF}\left|\left[\widetilde{K}_{-},\left[\widetilde{H}, \widetilde{K}_{-}\right]\right]\right| \mathrm{HF}\right\rangle}{N},
\end{gathered}
$$

where we have already indicated the use of the QBA which allows us to rewrite Eqs.(33) in matrix form

$$
\left(\begin{array}{cc}
A-\omega & B \\
B^{*} & A^{*}+\omega
\end{array}\right)\left[\begin{array}{l}
X \\
Y
\end{array}\right]=0 .
$$

Observing that $A=A^{*}$ and solving the eigenvalue problem, we find

$$
\omega=\sqrt{A^{2}-|B|^{2}} \quad \text { and } \quad X=\frac{B}{\omega-A} Y
$$

The problem is completely defined after fixing the overall constant relating $X$ and $Y$. In QBA we have

$$
\left\langle 0\left|\left[Q, Q^{\dagger}\right]\right| 0\right\rangle \simeq\left\langle\mathrm{HF}\left|\left[Q, Q^{\dagger}\right]\right| \mathrm{HF}\right\rangle=1
$$

which implies the following normalization condition

$$
|X|^{2}-|Y|^{2}=1
$$

By using the properties (28) and (29) we have that,

$$
\begin{aligned}
\left\langle 1\left|Q^{\dagger}\right| 0\right\rangle=1 & \Rightarrow \quad X\left\langle 1\left|\widetilde{K}_{+}\right| 0\right\rangle-Y\left\langle 1\left|\widetilde{K}_{-}\right| 0\right\rangle=\sqrt{N}, \\
\langle 1|Q| 0\rangle=0 & \Rightarrow \quad X^{*}\left\langle 1\left|\widetilde{K}_{-}\right| 0\right\rangle=Y^{*}\left\langle 1\left|\widetilde{K}_{+}\right| 0\right\rangle,
\end{aligned}
$$


and

$$
\left\langle 1\left|\widetilde{K}_{+}\right| 0\right\rangle=\sqrt{N} X^{*} \quad \text { and } \quad\left\langle 1\left|\widetilde{K}_{-}\right| 0\right\rangle=\sqrt{N} Y^{*} .
$$

Many terms of $\widetilde{H}$ do not contribute to the expectation value with respect to the RPA ground state. For this reason, instead of carring out the calculation with the full hamiltonian $\widetilde{H}$, we use an effective hamiltonian whose terms generate contributions different from zero and satisfy Eq. (28). The general expression of this hamiltonian, up to quadratic terms in $\widetilde{K}$, is given by $\underline{\underline{20}}$

$$
H_{\mathrm{RPA}}=E_{\mathrm{HF}}+\frac{1}{N}\left[A \widetilde{K}_{+} \widetilde{K}_{-}+\frac{1}{2}\left(B \widetilde{K}_{+}^{2}+B^{*} \widetilde{K}_{-}^{2}\right)\right]
$$

We can use the effective RPA hamiltonian (45) to calculate the energy of the RPA ground state

$$
\begin{aligned}
E_{\mathrm{RPA}} & =\left\langle 0\left|H_{\mathrm{RPA}}\right| 0\right\rangle \\
& =E_{\mathrm{HF}}+\frac{A\left\langle 0\left|\widetilde{K}_{+} \widetilde{K}_{-}\right| 0\right\rangle}{N}+\frac{\left\langle 0\left|\left(B \widetilde{K}_{+}^{2}+B^{*} \widetilde{K}_{-}^{2}\right)\right| 0\right\rangle}{2 N} \\
& =E_{\mathrm{HF}}+\frac{A\left\langle 0\left|\widetilde{K}_{+}\right| 1\right\rangle\left\langle 1\left|\widetilde{K}_{-}\right| 0\right\rangle}{N}+\frac{B\left\langle 0\left|\widetilde{K}_{+}\right| 1\right\rangle\left\langle 1\left|\widetilde{K}_{+}\right| 0\right\rangle+B^{*}\left\langle 0\left|\widetilde{K}_{-}\right| 1\right\rangle\left\langle 1\left|\widetilde{K}_{-}\right| 0\right\rangle}{2 N} \\
& =E_{\mathrm{HF}}+A|Y|^{2}+\frac{B Y X^{*}+B^{*} Y^{*} X}{2} \\
& =E_{\mathrm{HF}}+\left(A+\frac{|B|^{2}}{\omega-A}\right)|Y|^{2} \\
& =E_{\mathrm{HF}}-\omega|Y|^{2} .
\end{aligned}
$$

Considering the relation (39) and the normalisation of the $X$ and $Y$ (41), we have

$$
|Y|^{2}=\frac{A-\omega}{2 \omega}
$$

and we can write the energy of the RPA ground state as

$$
E_{0}^{\mathrm{RPA}}=E_{\mathrm{HF}}+\frac{\omega-A}{2}
$$

The evaluation of the the $A$ and $B$ coefficients is carried out in QBA. By using the commutation relations (17) between the $\widetilde{K}_{0, \pm}$ operators we obtain

$$
\left\langle\mathrm{HF}\left|\left[\widetilde{K}_{-},\left[\widetilde{K}_{0}, \widetilde{K}_{+}\right]\right]\right| \mathrm{HF}\right\rangle=\left\langle\mathrm{HF}\left|\left[\widetilde{K}_{-}, \widetilde{K}_{+}\right]\right| \mathrm{HF}\right\rangle=N
$$

Observing that

$$
\left[\widetilde{K}_{-},\left[\widetilde{K}_{0}^{2}, \widetilde{K}_{+}\right]=\frac{1}{2}\left[\widetilde{K}_{-},\left[\widetilde{K}_{+}^{2}, \widetilde{K}_{-}\right]\right]=-\frac{1}{2}\left[\widetilde{K}_{-},\left[\left\{\widetilde{K}_{+}, \widetilde{K}_{-}\right\}, \widetilde{K}_{+}\right]\right]\right.
$$


and

$$
\left\langle\tilde{k}, \widetilde{m}\left|\left[\widetilde{K}_{-},\left[\widetilde{K}_{0}^{2}, \widetilde{K}_{+}\right]\right]\right| \tilde{k}, \widetilde{m}\right\rangle=2\left[\tilde{k}(\tilde{k}+1)-3 \widetilde{m}^{2}\right]
$$

we find

$$
\begin{aligned}
\left\langle\mathrm{HF}\left|\left[\widetilde{K}_{-},\left[\widetilde{K}_{0}^{2}, \widetilde{K}_{+}\right]\right]\right| \mathrm{HF}\right\rangle & =-N(N-1) \\
\left\langle\mathrm{HF}\left|\left[\widetilde{K}_{-},\left[\left\{\widetilde{K}_{+}, \widetilde{K}_{-}\right\}, \widetilde{K}_{+}\right]\right]\right| \mathrm{HF}\right\rangle & =2 N(N-1) \\
\left\langle\mathrm{HF}\left|\left[\widetilde{K}_{-},\left[\widetilde{K}_{+}^{2}, \widetilde{K}_{-}\right]\right]\right| \mathrm{HF}\right\rangle & =-2 N(N-1)
\end{aligned}
$$

Putting together the previous results with the expression of the hamiltonian (19), we obtain

$$
\begin{aligned}
& A=\epsilon \cos \alpha+\frac{3}{2}(N-1)(V+W) \sin ^{2} \alpha-(N-1) W, \\
& B=-(N-1)(V+W) \frac{1+\cos ^{2} \alpha}{2}+(N-1) W .
\end{aligned}
$$

For $\alpha=\alpha_{\mathrm{HF}}$, we then find

$$
A= \begin{cases}\epsilon-(N-1) W & (\text { region I, }), \\ \frac{3(N-1)^{2}(V+W)^{2}-\epsilon^{2}}{2(N-1)(V+W)}-(N-1) W & (\text { region II), }\end{cases}
$$

and

$$
B= \begin{cases}-(N-1) V & (\text { region I) }, \\ -\frac{\epsilon^{2}+(N-1)^{2}(V+W)^{2}}{2(N-1)(V+W)}+(N-1) W & (\text { region II). }\end{cases}
$$

The behaviour of the RPA ground state energies, as a function of the strength $V$ of the interaction is shown in Figs. 10 and 2 by the blue dashed lines. For $W=0$, it is evident the improvement with respect to the HF results, especially in the region I. The value of the energy at the transition point between the two regions is

$$
-\frac{N+1}{2} \epsilon \text {. }
$$

For $W=V$ the agreement between RPA and exact results in the region I is excellent. In this case the value of the energy in the transition point is

$$
-\frac{4 N^{2}-1}{4(N-1)} \epsilon .
$$

The behaviour of the solutions for $W=V$ in the region II is remarkable. In this region we find for the RPA solution

$$
B=-\frac{\epsilon^{2}}{4(N-1) V} .
$$

For $4(N-1) V \gg \epsilon$ we have that $B \rightarrow 0$ and, consequently, due to the fact that $Y \rightarrow 0$, the value RPA energy $E_{0}^{\mathrm{RPA}}$ tends to that of the HF energy. 


\section{CONCLUSIONS}

The LMG many-fermion model, composed by two energy levels, is an ideal system where the many-body Schrödinger equation for the interacting particles can be solved without approximations. The comparison of these exact solutions with those obtained by using effective theories can give a measure of the validity of the latter ones.

We considered a hamiltonian containing two interacting terms. A first one, whose strength has been called $V$, scatters pairs of particles from one level to the other one, and a second term, whose strength is $W$, removes a particle from one level and put it on the other one.

In this article, we tested the validity of the HF and RPA theories in the description of the ground state of the system. In both cases, the solutions are characterised by two regions which depend on the strength of the interaction between the particles. The transition between the two regions is discontinuous. The discontinuity at the meeting point (which seems to suggest some sort of phase transition) is clearly an artefact of the effective theories, since the exact results do not present any discontinuity region. So, approximation methods could suggests anomalous behaviors which do not really happen in the real system. For strong interactions and large number of interacting particles the HF and RPA solutions approach the exact behavior of the system.

In the region I, characterised by relatively small values of the interaction, the HF energies are independent of $V$. This implies that for $W=0$, these energies are constant and show a remarkable discrepancy with respect to the exact result which become smaller when $V$ increases. The HF description of the exact results improves in the region II, and also when $W=V$.

The RPA solutions give a reasonable description of the exact results also in the region I for $W=0$, showing a large improvement with respect to the HF results. In all the cases we have considered, the RPA and HF solutions converge for large values of the interaction strength $V$. The solutions of the effective theories become closer to the exact ones when the number of the particles composing the system increases.

\footnotetext{
* gpco@le.infn.it

$\dagger$ deleo@ime.unicamp.br
} 
1 A. Messiah, Quantum Mechanics (North Holland, Amsterdam, 1961).

2 A. L. Fetter and J. D. Walecka, Quantum theory of many-particle systems (McGraw-Hill, S. Francisco, 1971).

3 W. Nolting, Fundamentals of many body physics (Springer-Verlag, Berlin, 2009).

4 M. Moshinsky, "How good is the Hartree-Fock approximation", Am. J. Phys. 36, 52-53 (1968).

5 M. Moshinsky, "How good is the Hartree-Fock approximation [Errata]", Am. J. Phys. 36, 663 (1968).

6 W. P. Latham Jr. and D. H. Kobe, "Comparison of the Hartree-Fock and maximum overlap orbitals for a simple model", Am. J. Phys. 36, 1258-1266 (1973).

7 Y. Nogami, M. Vallières, and W. van Dijk "HartreeFock approximation for the onedimensional helium atom",

Am. J. Phys. 44, 886-888 (1976).

8 A. Sauceda and W. van Dijk, "An exact Hartree solution of a onedimensional manybody problem with two and threebody interactions", Am. J. Phys. 46, 1195-1196 (1978).

9 H.J. Lipkin, N. Meshkov, and A.J. Glick, "Validity of many-body approximation methods for a solvable model [(I) Exact solutions and perturbation theory]", Nucl. Phys. 62, 188-198 (1965).

10 N. Meshkov, A.J. Glick, and H.J. Lipkin, "Validity of many-body approximation methods for a solvable model [(II) Linearization procedures]", Nucl. Phys. 62, 199-210 (1965).

11 A.J. Glick, H.J. Lipkin, and N. Meshkov, "Validity of many-body approximation methods for a solvable model [(III) Diagram summations]", Nucl. Phys. 62, 211-224 (1965).

12 D. R. Hartree, "The distribution of charge and current in an atom consisting of many electrons obeying Dirac's equations", Proc. Camb. Phyl. Soc. 25, 225-236 (1929).

13 V. Fock, "Näherungsmethode zur lösung des quantenmechanischen mehrkörperproblems", Z. Physik, 61, 126-148 (1930).

14 J. C. Slater Fock, "A siimplification of the Hartree-Fock method", Phys. Rev. 81, 385-390 (1950).

15 D. Bohm and D. Pines, "A Collective Description of Electron Interactions: III. Coulomb Interactions in a Degenerate Electron Gas", Phys. Rev. 92, 609-625 (1952).

16 D. J. Rowe, "Methods for calculating ground-state correlations of vibrational nuclei", Phys. Rev. 175, 1283-1292 (1968).

17 J. C. Parikh and D. J. Rowe, "Investigation of ground state correlations for a model hamiltonian 
of the nucleus", Phys. Rev. 175, 1293-1300 (1968).

18 K. Hagino and G. F. Bertsch, "Random-phase approximation approach to rotational symmetry restoration in a three-level Lipkin model", Phys. Rev. C 61, 024307-5 (2000).

19 W. H. Press, B. P. Flannery, S. A. Teukolsky, and W. T. Vetterling, Numerical recipes. The art of scientific computing (Cambridge University Press, Cambridge, 1986), p. 335-377.

20 P. Ring and P. Schuck, The nuclear many-body problem (Springer, Berlin, 1980). 


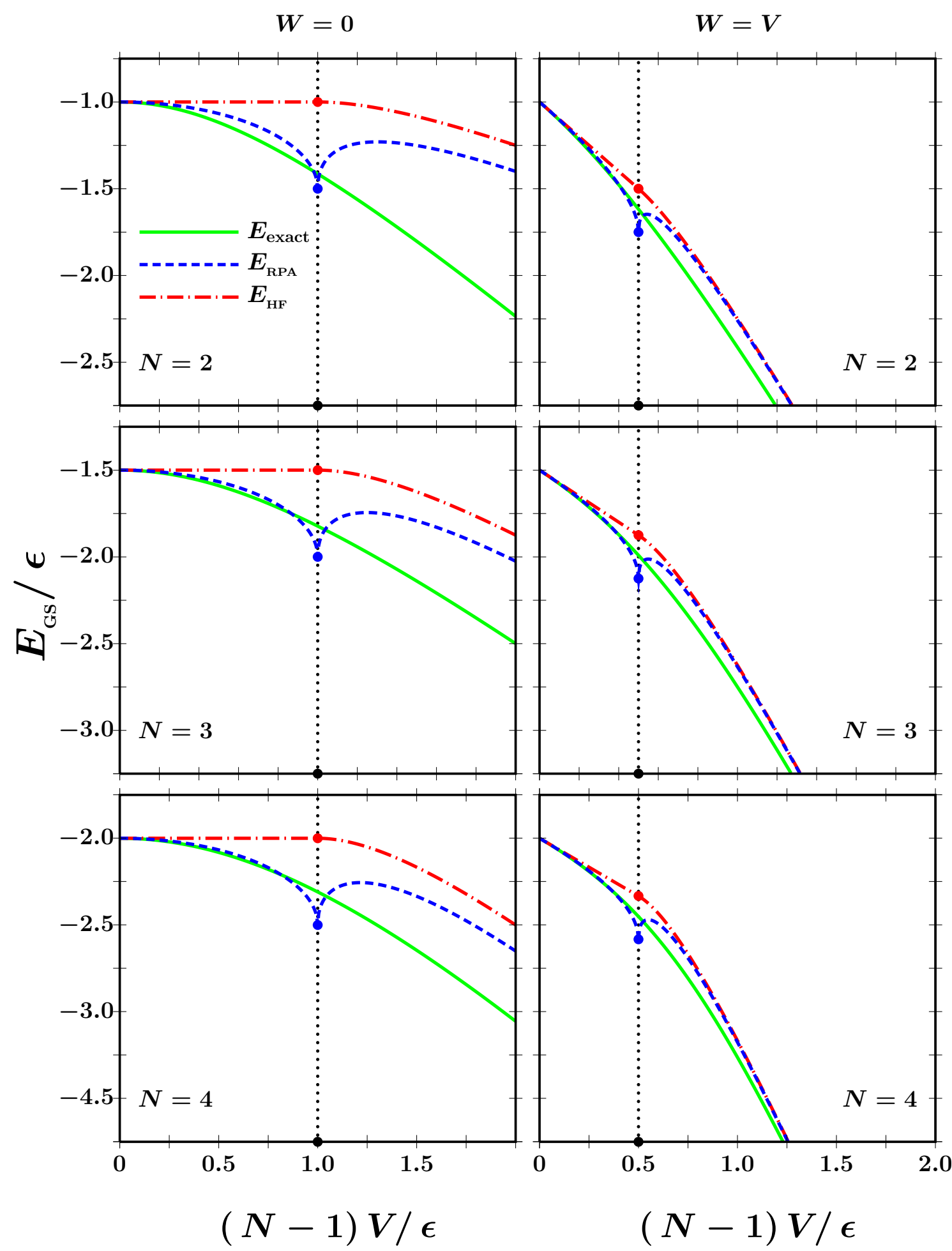

FIG. 1. Ground state energies as a function of the interaction strength $V$ for systems composed by $N=2,3$ and 4 particles. The full (green) lines indicate the solutions obtained without approximations. The dash-dotted (red) lines the results obtained with the HF model, and the dashed (blue) lines those obtained with the RPA approach. The left panels show the results for $W=0$, and the right panels those obtained by setting $W=V$. The blue and red thick dots emphasize the values of the HF and RPA energies in the discontinuity line. 


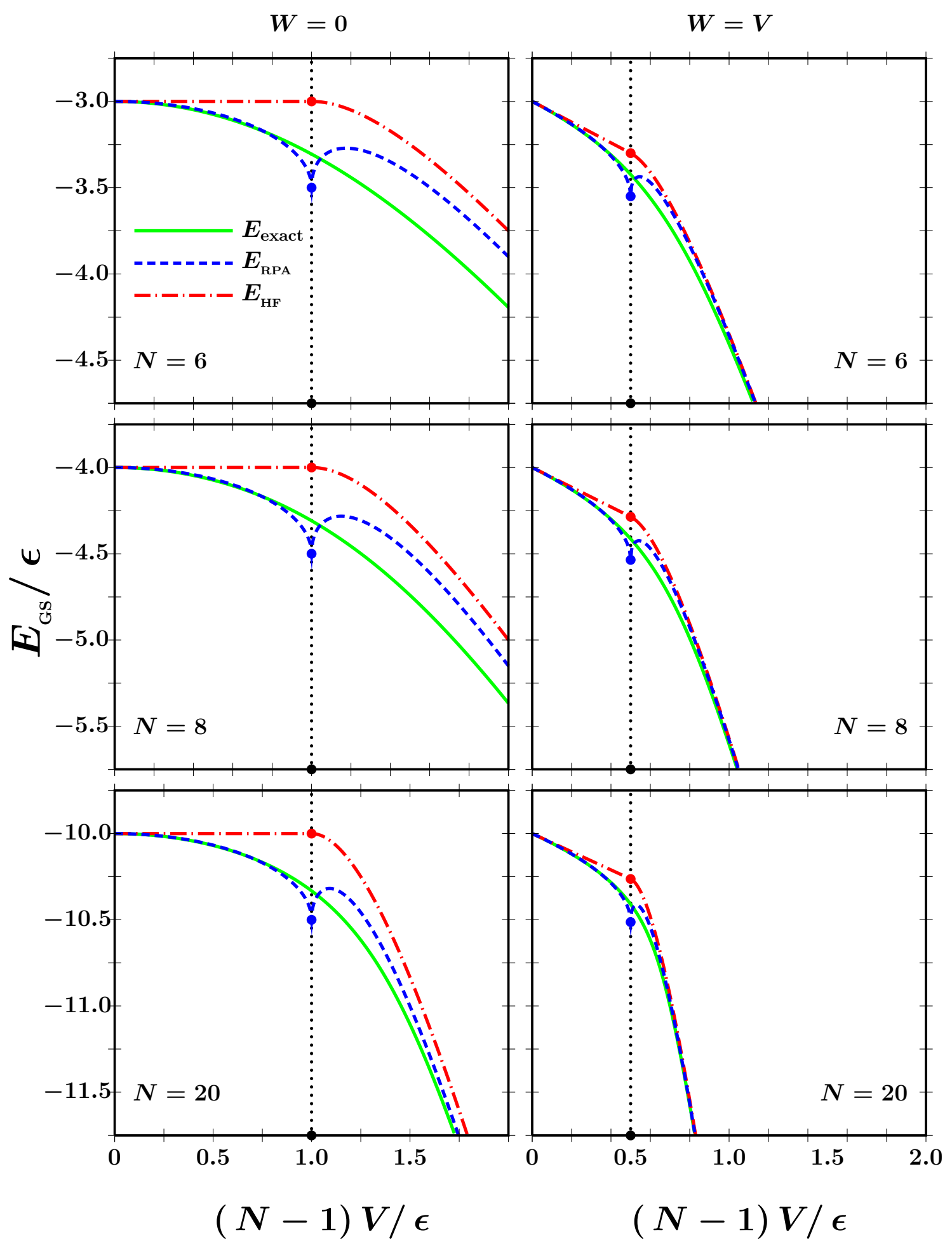

FIG. 2. The same as in Fig. 1 for $N=6,8$ and 20 . 